\title{
AVALIAÇÃO DA CONFIABILIDADE DE PONTES EM CONCRETO PROTENDIDO SOB SOLICITAÇÕES NORMAIS
}

\author{
Evaluation of The Reliability of Prestressed Concrete Bridges Under Normal Loads
}

\author{
Silvia Juliana Sarmiento Nova ${ }^{1}$, Maria Cecilia Amorim Teixeira da Silva ${ }^{2}$
}

Recebido em 16 de dezembro de 2016; recebido para revisão em 04 de abril de 2017; aceito em 17 de maio de 2017; disponível on-line em 18 de agosto de 2017.

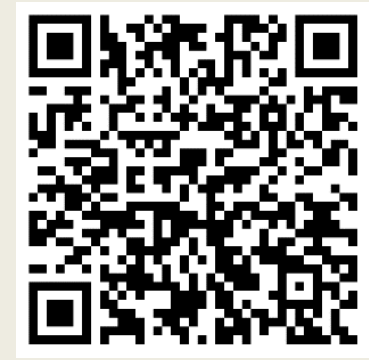

PALAVRAS CHAVE:

Confiabilidade;

Pontes;

Probabilidade de falha;

Concreto protendido;

Solicitações normais.

\section{KEYWORDS:}

Reliability;

Bridges;

Probability of failure;

Prestressed concrete;

Normal loads.

\begin{abstract}
RESUMO: O presente trabalho consiste em sistematizar um procedimento para avaliação da confiabilidade estrutural de pontes de concreto protendido, com base no índice de confiabilidade $\beta$, e a correspondente probabilidade de falha $P_{f} . O$ Método de Monte Carlo é utilizado no processo de simulação das variáveis aleatórias, tomadas como sendo as resistências dos materiais, algumas das propriedades geométricas e o carregamento aplicado à estrutura. Como ferramenta para análise estrutural, é utilizado um programa computacional comercial. Os valores obtidos pelo método de simulação são validados através do método analítico FORM, e realiza-se também uma análise de sensibilidade. O procedimento é aplicado a uma ponte de concreto protendido de viga-e-laje. Com base nos resultados alcançados na análise desenvolvida conclui-se que o procedimento sistematizado, o qual utiliza um método de simulação, pode ser utilizado para avaliar a confiabilidade de uma ponte em concreto protendido de uma forma mais prática e efetiva. A análise de sensibilidade permitiu identificar as variáveis que apresentam maior influência na avaliação da segurança da estrutura.
\end{abstract}

ABSTRACT: This paper is proposed to systematize the structural reliability evaluation procedure of prestressed concrete bridges, based on the reliability index 6 , and the corresponding probability of failure $\mathrm{P}_{\mathrm{f}}$. The Monte Carlo method is used to simulate design variables, taken as the resistances of the materials, some geometrical properties and the loads applied to the structure. As a tool for structural analysis, a commercial computer program is used. The values obtained by simulation method are validated by the analytical method FORM, and a sensitivity analysis is carried out. The procedure is applied to a deck-girder prestressed concrete bridge. Based on the results obtained, it is concluded that the procedure, which is based on a simulation method, can be used to evaluate the reliability of a prestressed concrete bridge in a more practical and effective way. The sensitivity analysis identified the variables with the greatest influence on the assessment of the safety of the structure.

* Contato com os autores:

${ }^{1}$ e-mail: sjsarmiento.n@gmail.com (S. J. S. Nova )

Engenheira Civil, Mestranda do Curso de Pós-graduação em Engenharia Civil da Faculdade de Engenharia Civil, Arquitetura e Urbanismo FEC-UNICAMP.

2e-mail: cecilia@fec.unicamp.br (M. C. A. T. Silva )

Engenheira Civil, Doutora, Professora da Faculdade de Engenharia Civil, Arquitetura e Urbanismo, Departamento de Estruturas, FEC-UNICAMP. 


\section{INTRODUÇÃO}

As estruturas em concreto protendido vem sendo implementadas na construção civil em uma grande quantidade de obras, destacando-se principalmente pontes e viadutos. Atualmente, o concreto protendido é amplamente utilizado em estruturas como edificações, pistas de aeroportos, reservatórios de água, silos, entre outras. Tendo em vista que o conjunto representativo de estruturas em concreto protendido é amplo, avaliar a segurança desse tipo de estrutura passou a ser um assunto de importância.

Trabalhos recentemente desenvolvidos na área de confiabilidade apontam para um aspecto que merece destaque: "por razões econômicas e de segurança, é imperativo assegurar que as pontes, como elementos vitais da infra-estrutura de transporte terrestre, se mantenham em condição aceitável e com elevado nível de confiabilidade" (Jacinto, 2011). Na determinação da confiabilidade existe uma quantidade de incertezas causadas pela aleatoriedade de alguns parâmetros, as quais devem ser consideradas no cálculo da probabilidade de falha de uma estrutura. Parte dessas incertezas são causadas por erros humanos que afetam o desempenho e, portanto, a segurança da estrutura. Segundo Beck (2006), existem modelos empíricos que representam as incertezas decorrentes da ação humana.

Formas de implementação de métodos probabilísticos tem passado por um desenvolvimento importante nos últimos anos: tanto a probabilidade quanto a estatística se converteram em ferramentas essenciais para a avaliação da incerteza. Especialmente na Engenharia Estrutural, a confiabilidade tem sido estabelecida como base no desenvolvimento dos códigos de projeto. No caso específico da avaliação da integridade das estruturas em concreto protendido, existem imprecisões nas variáveis envolvidas no projeto, que fazem indispensável o uso de uma abordagem probabilística.

Tendo em vista que as pontes constituem uma proporção significativa da rede viária, juntamente com o crescente uso da protensão no
Brasil e a carência de análise sobre os diversos parâmetros relacionados com o projeto para segurança estrutural utilizados nas normas atuais, o estudo da confiabilidade de pontes em concreto protendido torna-se um tema de relevância.

\section{OBJETIVOS}

Estabelece-se como objetivo principal deste trabalho sistematizar um procedimento que permita avaliar a segurança dos elementos estruturais que compõem a superestrutura de pontes em concreto protendido, através do cálculo do índice de confiabilidade $\beta$ e a probabilidade de falha $P_{f}$ da estrutura.

Além de descrever o procedimento sistematizado, o trabalho tem como finalidade avaliar a segurança de uma ponte de concreto protendido em um sistema viga-e-laje que foi estabelecida como estudo de caso e, através dos resultados alcançados, identificar os parâmetros que apresentam maior influência na avaliação da segurança da estrutura por meio de uma análise de sensibilidade.

\section{CONTEXTUALIZAÇÃO}

Trabalhos relacionados com a avalição da segurança de pontes em concreto protendido vem sendo desenvolvidos por diversos pesquisadores ao longo do tempo, destacando-se Chandrasekar e Dayaraatnam (1975), Tabsh e Nowak (1991), AlHarthy e Frangopol (1994), Nowak et al. (2001), Biondini et al. (2004), Cheng et al. (2007), e San Martins (2014).

Dos estudos relacionados com a confiabilidade estrutural, observa-se que uma das formas mais comuns na avaliação do nível de segurança é através do cálculo do índice de confiabilidade $\beta$ por meio do qual se obtém a correspondente probabilidade de falha $P_{f}$.

$\mathrm{Na}$ avaliação da segurança estrutural estabelece-se como fundamental determinar as Funções Estado Limite, pois por meio delas é definido o desempenho da estrutura em função de um conjunto de variáveis aleatórias. $\mathrm{O}$ cálculo do 
índice de confiabilidade $\beta$ é, a princípio, estabelecido para o sistema que representa a estrutura. De forma simplificada, contudo, é possível calcular o índice de confiabilidade para um dos elementos principais da estrutura analisada. No caso de uma ponte composta por um sistema vigae-laje, assume-se a viga protendida como sendo esse elemento principal. Nesse caso, as Funções Estado Limite são definidas considerando-se os três casos básicos de falha de uma viga protendida os quais, segundo Akgül e Frangopol (2004), são: flexão positiva na seção crítica, tensão à tração do concreto, e flexão negativa na seção crítica.

As variáveis que comumente são consideradas como aleatórias no estudo da confiabilidade de vigas em concreto protendido são mencionadas em estudos desenvolvidos por AlHarthey e Frangopol (1994), Nowak e Collins (2000), Akül e Frangopol (2004), Steinberg (2010) e Rocha et. al (2015), juntamente com a distribuição de probabilidade adotada para cada variável aleatória. Também o código elaborado pela JCSS (2000) fornece diretrizes importantes para a escolha dessas distribuições e para o cálculo dos parâmetros probabilísticos.

Os métodos comumente utilizados na avaliação da segurança estrutural podem ser divididos em: métodos analíticos e métodos de simulação. Dos métodos analíticos é largamente conhecido o método de confiabilidade de primeira ordem FORM, o qual é baseado na Teoria da Confiabilidade e foi implementado por diferentes pesquisadores tais como Al-Harthy e Frangopol (1994), Agrawak e Bhattacharya (2010), Abejide (2014), Halhalli (2014), e Rocha et al. (2015); alguns desses autores utilizaram para essa implementação o procedimento de Rackwitz e Fiessler (1978). Por outro lado, o método de Monte Carlo é um método de simulação usualmente implementado em análises de confiabilidade, e foi utilizado em trabalhos como Biondini et al. (2004) e Steinberg (2010) no caso de estruturas protendidas.

Juntamente com a confiabilidade estrutural, surgiu a necessidade de identificar aqueles parâmetros que manifestam um efeito significativo na segurança de uma estrutura. Para conhecê-los, diversos autores têm empregado análises de sensibilidade. Gardner et al. (1981) recomendaram o uso de coeficientes de correlação derivados de simulações de Monte Carlo como uma forma razoável de classificar as variáveis aleatórias de acordo com sua contribuição na análise. Além de coeficientes de correlação existem outras técnicas de análise de sensibilidade: algumas delas foram definidas por Hamby (1994), como por exemplo o uso do índice de importância que é derivado do método FORM.

\section{METODOLOGIA}

No presente artigo foi proposto um procedimento baseado na Teoria da Confiabilidade para avaliar a segurança de pontes de concreto protendido em um sistema viga-e-laje, levando-se em consideração os casos mais prováveis de falha da estrutura. O procedimento foi dividido em três etapas: definição das Funções Estado Limite, escolha das variáveis aleatórias, e cálculo da probabilidade de falha utilizando-se duas abordagens.

O procedimento de avaliação da segurança proposto foi aplicado a uma ponte de um único vão, composta por 4 vigas protendidas de 26 metros de comprimento espaçadas a cada 2,1 metros, e por uma laje de 8 metros de largura.

\subsection{DEFINIÇÃO DA FUNÇÃO ESTADO LIMITE}

A Função Estado Limite representa basicamente o efeito de uma ação $S$ que é suportada por uma resistência $R$, ambas descritas por funções de densidade de probabilidade conhecidas.

A determinação das Funções Estado Limite para pontes de concreto protendido em um sistema viga-e-laje foi derivada segundo os requerimentos estabelecidos na AASHTO LRFD Bridge Design Specifications (2012). Como o caso analisado no presente trabalho consiste em uma ponte de um único vão e as vigas encontram-se bi-apoiadas, os casos mais críticos de falha a serem considerados foram reduzidos a: flexão positiva no meio do vão e tensão à tração do concreto. 


\subsubsection{Flexão Positiva no Meio do Vão}

A função estado limite $G_{1}$ para uma viga submetida à flexão é definida através da Equação 1.

$$
\mathrm{G}_{1}=\mathrm{M}_{\mathrm{u}}-\left(M_{D C}+M_{D W}+M_{L L+I M}\right) \quad \text { Eq. [1] }
$$

\section{Em que:}

$\boldsymbol{M}_{u}$ : momento último resistente $(\mathrm{kN} \cdot \mathrm{m})$;

$D C$ : ação do peso próprio dos elementos estruturais $(\mathrm{kN} / \mathrm{m})$;

DW: ação do peso próprio dos elementos não estruturais $(\mathrm{kN} / \mathrm{m})$;

$M_{D C}$ : momento devido à ação $D C(\mathrm{kN} \cdot \mathrm{m})$;

$M_{D W}$ : momento devido à ação $D W(\mathrm{kN} . \mathrm{m})$;

LL: ação veicular $(\mathrm{kN} / \mathrm{m})$;

IM: carga de impacto;

$M_{L L+I M}$ : momento devido à ação $L L$ acrescida da carga de impacto IM $(\mathrm{kN} \cdot \mathrm{m})$.

O momento último resistente é calculado dependendo do comportamento estrutural que apresenta a viga. Assumindo-se o comportamento da viga como retangular, definiu-se o momento resistente através da Equação 2.

$$
\mathrm{M}_{\mathrm{u}}=A_{p s} f_{p s}\left(d_{p}-\frac{a}{2}\right)
$$

sendo,

$$
\begin{gathered}
a=\beta_{1} c \\
c=\frac{A_{p s} f_{p u}}{0,85 f^{\prime}{ }_{c} \beta_{1} b+k A_{p s} \frac{f_{p u}}{d_{p}}} \\
f_{p s}=f_{p u}\left(1-k \frac{c}{d_{p}}\right) \\
k=2 \cdot\left(1,04-\frac{f_{p y}}{f_{p u}}\right)
\end{gathered}
$$

Em que:

$f_{p s}$ : tensão média no aço de protensão $\left(\mathrm{kN} / \mathrm{m}^{2}\right)$;

$\boldsymbol{A}_{p s}$ : área transversal do cabo de protensão $\left(\mathrm{m}^{2}\right)$; $\boldsymbol{d}_{p}$ : distância entre a fibra de compressão extrema até o centróide dos cabos de protensão $(m)$;

$\boldsymbol{b}$ : largura da alma da viga $(\mathrm{m})$;

$\boldsymbol{a}$ : profundidade equivalente do bloco de tensão (m);

c: distância a partir do extremo da fibra de compressão até o eixo neutro $(\mathrm{m})$;

$\boldsymbol{\beta}_{1}$ : fator do bloco de tensão;

$f^{\prime}$ : resistência específica a compressão do concreto (aos 28 dias) $\left(\mathrm{kN} / \mathrm{m}^{2}\right)$;

$f_{p y}$ : resistência à fluência do aço de protensão $\left(\mathrm{kN} / \mathrm{m}^{2}\right)$;

$f_{p u}$ : é a resistência específica à tração do aço de protensão $\left(\mathrm{kN} / \mathrm{m}^{2}\right)$.

O momento advindo da carga variável foi calculado através da Equação 7.

$$
\mathrm{M}_{\mathrm{LL}+\mathrm{IM}}=\left(M_{v e} \cdot(1+I M)+M_{c a}\right) \cdot D F M \text { Eq. [7] }
$$

\section{Em que:}

DFM: fator de distribuição do momento de flexão; $M_{v e}$ : Momento devido ao peso do veículo de projeto $(\mathrm{kN} \cdot \mathrm{m})$;

$\boldsymbol{M}_{\boldsymbol{c a}}$ : Momento devido a uma carga acidental distribuída de carril $(\mathrm{kN} \cdot \mathrm{m})$.

O fator DFM é obtido segundo as especificações da AASHTO LRFD 4.6.2.2.2, e sua finalidade é distribuir os momentos advindos das ações variáveis nas vigas que compõem a estrutura. Este fator depende do tipo de estrutura que está sendo avaliada.

No cálculo dos momentos gerados no elemento devido ao veículo de projeto e à carga distribuída devem-se implementar as linhas de influência para os casos mais críticos. Para o caso de uma viga bi-apoiada, foram utilizadas as Equações 8 e 9.

$$
\begin{aligned}
M_{D C} & =\frac{D C \cdot L^{2}}{8} \\
M_{D W} & =\frac{D W \cdot L^{2}}{8}
\end{aligned}
$$

Em que:

$L$ : comprimento da viga protendida (m).

Finalmente foi obtida a Equação 10 que define a primeira Função Estado Limite. 


$$
\mathrm{G}_{1}=A_{p s} f_{p s}\left(d_{p}-\frac{a}{2}\right)-M_{D C}-M_{D W}-\left(M_{v e} \cdot(1+I M)+M_{c a}\right) \cdot D F M
$$

\subsubsection{Tensão à tração do concreto}

O estado limite de serviço de uma viga em concreto protendido pode ser avaliado com base nas tensões à tração e à compressão limite do concreto, na tensão à tração limite no aço de protensão, e na deformação limite à flexão.

No presente trabalho foi considerada a função estado limite $G_{2}$ para a tensão à tração do concreto de uma viga protendida, mostrada na Equação 11.

$$
\mathrm{G}_{2}=16 \sqrt{f_{c}^{\prime}}-\left(f_{p b}+f_{D C}+f_{D W}+f_{L L+I M}\right)
$$

Em que:

$f^{\prime}$ : resistência especifica à compressão do concreto (aos 28 dias) $\left(\mathrm{kN} / \mathrm{m}^{2}\right)$;

$f_{p b}$ : tensão à flexão na fibra inferior da viga devido à força de protensão $\left(\mathrm{kN} / \mathrm{m}^{2}\right)$;

$f_{D C}$ : tensão à flexão na fibra inferior da viga devido ao peso próprio dos elementos estruturais $\left(\mathrm{kN} / \mathrm{m}^{2}\right)$; $f_{D w}$ : tensão à flexão na fibra inferior da viga devido ao peso próprio dos elementos não estruturais $\left(\mathrm{kN} / \mathrm{m}^{2}\right)$;

$f_{L L+I M}$ : tensão à flexão na fibra inferior da viga devido à carga veicular incluindo o fator de impacto $\left(\mathrm{kN} / \mathrm{m}^{2}\right)$.

A tensão devida à força de protensão $f_{p b}$ pode ser calculada através das Equação 12.

$$
f_{p b}=-\frac{\sum P_{\text {final }}}{A_{G}}-\frac{\sum P_{\text {final }} \cdot e_{i}}{S_{G i}}
$$

sendo,

$$
\begin{gathered}
S_{G i}=\frac{y_{G i}}{I_{G}} \quad \text { Eq. [13] } \quad f_{L L+I M}=\frac{M_{L L+I M}}{S_{G i}} \\
\mathrm{G}_{2}=16 \sqrt{f^{\prime}{ }_{c}}-\left(-\frac{\sum P_{\text {final }}}{A_{G}}-\frac{\sum P_{\text {final }} \cdot e_{i}}{S_{G i}}+\frac{M_{D C}}{S_{G i}}+\frac{M_{D W}}{S_{G i}}+\frac{M_{L L+I M}}{S_{G i}}\right)
\end{gathered}
$$

$$
f_{D C}=\frac{M_{D C}}{S_{G i}}
$$

\section{Em que:}

$\boldsymbol{P}_{\text {final: }}$ força da protensão após as perdas totais $(\mathrm{kN})$; $\boldsymbol{e}_{i}$ : excentricidade individual de cada cabo da armadura de protensão $(\mathrm{m})$;

$S_{G i}$ : módulo de seção na fibra inferior da viga $\left(1 / \mathrm{m}^{3}\right)$; $y_{G i}$ : distância entre a parte inferior da viga e o eixo neutro $(\mathrm{m})$.

A força final de protensão é a força aplicada nos cabos descontadas as perdas e é definida na Equação 14.

$$
P_{\text {final }}=P_{\text {inicial }}-\Delta f_{p T} A_{p s}
$$

Em que:

$\Delta f_{p T}$ : perdas totais da força de protensão, as quais incluem perdas devidas ao atrito $\left(\Delta f_{p F}\right)$, à acomodação da ancoragem $\left(\Delta f_{p A}\right)$, ao encurtamento e ao alongamento elástico $\left(\Delta f_{p E S}\right)$, e as perdas progressivas causadas pela retração e fluência do concreto e pela relaxação do aço $\left(\Delta f_{p L T}\right)\left(\mathrm{kN} / \mathrm{m}^{2}\right)$.

Nas Equações 15 a 17 mostram-se as tensões que são provocadas pelas ações permanentes e variáveis que solicitam a estrutura. Substituindo-as na Equação 11, a Função Estado Limite $G_{2}$ fica definida pela Equação 18.

$$
f_{D W}=\frac{M_{D W}}{S_{G i}}
$$




\subsection{VARIÁVEIS ALEATÓRIAS}

As variáveis aleatórias consideradas em cada Função Estado Limite, foram definidas segundo as referências citadas no item 3 e encontram-se resumidas na Tabela 1 e ilustradas na Figura 1.

\subsubsection{Distribuições e parâmetros}

Para cada variável aleatória, adotou-se a distribuição de probabilidade e assumiu-se o coeficiente de variação $V_{x}$, ambos estabelecidos pelos autores referenciados na Tabela 2 .

Os parâmetros de cada distribuição a ser utilizada foram obtidos pelas Equações 19 a 22. Foram escolhidos como valores característicos $X_{k}$ os valores nominais ou de projeto.

1. Para distribuição normal:

$$
\mu_{x}=\frac{x}{1-u \cdot V_{x}}
$$

\section{TABELA 1: Variáveis aleatórias para as Funções Estado Limite.}

\begin{tabular}{cc}
$\mathbf{G}_{\mathbf{1}}$ & $\mathbf{G}_{\mathbf{2}}$ \\
\hline$A_{p s}$ & $A_{p s}$ \\
\hline$y_{b s}$ & $y_{b s}$ \\
\hline$b$ & $y_{G c}$ \\
\hline$f_{c}^{\prime}$ & $f_{c}^{\prime}$ \\
\hline$f_{p u}$ & $f_{p u}$ \\
\hline$h_{\text {viga }}$ & $P_{\text {inicial }}$ \\
\hline$D C$ & $D C$ \\
\hline$M_{\text {ve }}$ & $M_{v e}$ \\
\hline
\end{tabular}

FONTE: Autoria Própria.

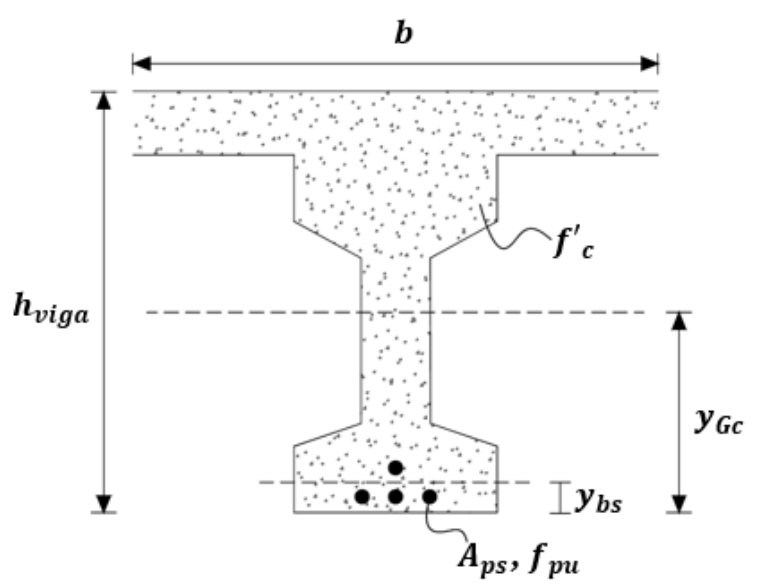

FIGURA 1: Representação gráfica das variáveis aleatórias.

FONTE: Autoria própria.

$$
\sigma_{x}=V_{x} \cdot \mu_{x}
$$

2. Para distribuição Log-Normal:

$$
\begin{gathered}
\mu_{x}=e^{\ln (x)+u \sqrt{\ln \left(V_{x}^{2}+1\right)}+\frac{1}{2}} \ln \left(V_{x}^{2}+1\right) \\
\sigma_{x}=V_{x} \cdot \mu_{x}
\end{gathered}
$$

Em que:

$\boldsymbol{\mu}_{\boldsymbol{x}}$ : média da variável em análise;

$\sigma_{x}$ : desvio padrão da variável em análise;

$\boldsymbol{V}_{\boldsymbol{x}}$ : coeficiente de variação;

$u$ : variável normal reduzida;

$\boldsymbol{x}$ : variável aleatória em análise.

TABELA 2: Distribuição e coeficiente de variação para cada variável aleatória.

\begin{tabular}{cccl} 
Variável & Distribuição & $\boldsymbol{V}_{\boldsymbol{x}}$ & \multicolumn{1}{c}{ Referência } \\
\hline $\boldsymbol{A}_{\boldsymbol{p} \boldsymbol{s}}$ & Normal & 0,0125 & Akgül e Frangopol (2004) \\
\hline $\boldsymbol{y}_{\boldsymbol{b s}}$ & Normal & 0,08 & Al-Harthy e Frangopol (1994), Steinberg (2010) \\
\hline $\boldsymbol{y}_{\boldsymbol{G} \boldsymbol{c}}$ & Normal & 0,009 & Al-Harthy e Frangopol (1994), Steinberg (2010) \\
\hline $\boldsymbol{b}$ & Normal & 0,004 & Al-Harthy e Frangopol (1994), Steinberg (2010) \\
\hline $\boldsymbol{f}_{\boldsymbol{c}}^{\prime}$ & Log-Normal & 0,15 & Al-Harthy e Frangopol (1994), Nowak e Collins (2000) \\
\hline $\boldsymbol{f}_{\boldsymbol{p u}}$ & Log-Normal & 0,025 & Al-Harthy e Frangopol (1994) \\
\hline $\boldsymbol{h}_{\boldsymbol{v i g a}}$ & Normal & 0,008 & Al-Harthy e Frangopol (1994), Steinberg (2010) \\
\hline $\boldsymbol{P}_{\text {inicial }}$ & Normal & 0,05 & Akgül e Frangopol (2004) \\
\hline $\boldsymbol{D C}$ & Normal & 0,1 & Al-Harthy e Frangopol (1994) \\
\hline $\boldsymbol{M}_{\boldsymbol{v e}}$ & Gumbel & 0,25 & Al-Harthy e Frangopol (1994), Steinberg (2010)
\end{tabular}


Para as variáveis $A_{p s}, h_{v i g a}, y_{b s}$ e $y_{G c}$ foi adotado como valor da média $\mu_{k}$ o valor nominal ou valor de projeto, como estabelecido por Steinberg (2010) e por Al-Harthey e Frangopol (1994).

A média $\mu_{b}$ da variável $b$ foi adotada segundo a Equação 23 (Steinberg, 2010):

$$
\mu_{b}=b_{k}+0,004
$$

Em que:

$\mu_{b}$ : valor da média da variável aleatória $b(\mathrm{~m})$;

$\boldsymbol{b}_{\boldsymbol{k}}$ : valor nominal ou característico da variável em análise $(\mathrm{m})$.

Para as ações permanentes e os efeitos das ações variáveis que atuam na estrutura, nesse caso o peso próprio da estrutura DC e o momento devido ao veículo de projeto $\mathrm{M}_{\mathrm{ve}}$, as médias foram calculadas utilizando-se as Equações 24 e 25, respectivamente, segundo o estabelecido por Al-Harthy e Frangopol (1994).

$$
\begin{aligned}
& \mu_{D C}=D C_{k} \cdot 1,05 \\
& \mu_{M_{v e}}=M_{v e k} \cdot 0,89
\end{aligned}
$$

Em que:

$\mu_{D C}$ : valor da média da variável aleatória $D C(\mathrm{kN} / \mathrm{m})$; $D C_{k}$ : valor nominal ou característico da vaiável em análise $(\mathrm{kN} / \mathrm{m})$;

$\mu_{M v e:}$ valor da média da variável aleatória $M_{v e}$ (kN.m);

$M_{\text {vek: }}$ valor nominal ou característico da vaiável em análise $(\mathrm{kN} \cdot \mathrm{m})$.

Para as variáveis $f_{c}^{\prime}, f_{p u}$ e $P_{\text {inicial }}$ as médias foram calculadas utilizando-se as Equações 19 e 21, as quais dependem da distribuição de probabilidade. O desvio-padrão de cada uma delas foi obtido através da expressão 20. Na Tabela 3 são apresentados os valores de projeto, a média e o desvio-padrão de cada variável aleatória.

\subsection{CÁLCULO DA PROBABILIDADE DE FALHA}

O cálculo da probabilidade de falha foi realizado através de duas abordagens: utilizando-se o método de simulação Monte Carlo e utilizando-se o Método de Confiabilidade de Primeira Ordem FORM. Os valores obtidos pelo FORM tiveram como finalidade ratificar os resultados obtidos pela simulação por Monte Carlo.

\subsubsection{Método de Simulação Monte Carlo}

O método de simulação Monte Carlo foi aplicado através do software MATLAB, o qual permitiu gerar um número $\mathrm{N}$ de valores randômicos para cada variável aleatória, seguindo uma determinada função de distribuição de probabilidade. Com esses valores, as Funções Estado Limite foram avaliadas $\mathrm{N}$ vezes, de tal forma que pudesse ser calculada a probabilidade de falha através da Equação 26. O número $\mathrm{N}$ de simulações implementado foi de 10.000.000, o que significa que cada variável aleatória obteve 10.000.000 de valores randômicos.

\section{TABELA 3: Parâmetros probabilísticos de cada variável aleatória.}

\begin{tabular}{cccc} 
Variável & $\boldsymbol{X}_{\boldsymbol{k}}$ & $\boldsymbol{\mu}_{\boldsymbol{k}}$ & $\boldsymbol{\sigma}_{\boldsymbol{k}}$ \\
\hline $\boldsymbol{A}_{\boldsymbol{p} s}$ & 0,00276 & 0,00276 & 0,0000345 \\
\hline $\boldsymbol{y}_{\boldsymbol{b s}}$ & 0,103 & 0,103 & 0,0082 \\
\hline $\mathbf{Y}_{G c}$ & 0,95 & 0,95 & 0,009 \\
\hline $\boldsymbol{b}$ & 1,627 & 1,63 & 0,006 \\
\hline $\boldsymbol{f}_{\boldsymbol{c}}^{\prime}$ & 35000 & 45234,14 & 6785,12 \\
\hline $\boldsymbol{f}_{\boldsymbol{p u}}$ & 1890000 & 1969949,30 & 49248,73 \\
\hline $\boldsymbol{h}_{\text {viga }}$ & 1,25 & 1,25 & 0,010 \\
\hline $\boldsymbol{P}_{\text {inicial }}$ & 3917,40 & 4268,49 & 213,42 \\
\hline $\boldsymbol{D C}$ & 15,55 & 16,33 & 1,63 \\
\hline $\boldsymbol{M}_{\boldsymbol{v e}}$ & 1762,4 & 1575,59 & 393,90 \\
\hline & & & FONTE: Autoria Própria.
\end{tabular}




$$
\begin{aligned}
& P_{f}=\frac{n}{N} \quad \text { Eq. [26] }
\end{aligned}
$$

Em que:

$n$ : número de vezes em que $G(x)$ alcança um valor menor ou igual a zero.

$\mathbf{N}$ : número total de simulações.

O método considerou as variáveis aleatórias definidas no item 4.2, seguindo as funções de distribuição de probabilidade estabelecidas no mesmo item com seus respectivos parâmetros.

O valor do índice de confiabilidade $\beta$, é definido pela Equação 27.

$$
\beta=-\phi^{-1}\left(P_{f}\right)
$$

Em que:

$\boldsymbol{\beta}$ : índice de confiabilidade;

$\boldsymbol{P}_{f: \text { probabilidade de falha. }}$

\subsubsection{Método de Confiabilidade de Primeira Ordem FORM}

Como foi mencionado, para validar os valores do índice de confiabilidade e da probabilidade de falha alcançados na simulação Monte Carlo, utilizou-se o método FORM. Esse método pode ser implementado por meio do procedimento iterativo de Rackwitz-Fiessler, o qual está baseado na Teoria da Confiabilidade, e exige que a distribuição de probabilidade de cada variável envolvida seja conhecida.

O procedimento de Rackwitz-Fiessler busca obter o valor do índice de confiabilidade assim como o valor de cada variável aleatória no ponto em que é achada a densidade de probabilidade (Most Probability Point). Nesse procedimento é requerido que um ponto inicial seja escolhido para começar o processo iterativo. Segundo Nowak e Collins (2000) é recomendável escolher as médias de cada variável como valores iniciais desse ponto de partida. Sabendo-se que a análise deve ser feita na superfície de falha, faz-se a função estado limite $G(x)$ igual a zero e resolve-se a equação para uma das variáveis aleatórias previamente escolhida. No presente trabalho a variável selecionada foi o momento devido ao veículo de projeto, $M_{\text {ve }}$.

\subsection{ANÁLISE DE SENSIBILIDADE}

Tendo em vista aprimorar o modelo de avaliação da segurança da ponte em estudo, optouse por realizar a análise de sensibilidade das variáveis aleatórias envolvidas. A aplicação dessa técnica permite inicialmente determinar as incertezas que influenciam no colapso ou no evento de falha em análise, e em seguida localizar aquelas variáveis que apresentam maior sensibilidade na resposta, levando a uma diminuição da quantidade de variáveis aleatórias a serem consideradas e consequentemente à redução do custo computacional.

Como foi ressaltado no item 3, existem diversos métodos que podem avaliar a sensibilidade dos parâmetros envolvidos numa análise de confiabilidade. No presente trabalho foram utilizados dois métodos, com o fim de comparar as respostas obtidas.

O primeiro método consiste em calcular um índice chamado de Índice de Importância $I_{i}$, o qual está em função do fator de sensibilidade e é definido pela Equação 28. O fator de sensibilidade foi obtido na implementação do método FORM.

$$
I_{i}=\alpha_{i}^{2}
$$

Em que:

$\boldsymbol{\alpha}_{\mathrm{i}}$ : fator de sensibilidade de cada variável.

O segundo método utilizado consiste em calcular o coeficiente de Pearson, o qual é um coeficiente que determina a correlação entre duas variáveis.

O coeficiente de Pearson é definido pela Equação 29:

$$
r=\frac{\sum_{i=1}^{n}\left(x_{i}-\mu_{x}\right)\left(y_{i}-\mu_{y}\right)}{\left[\sum_{i=1}^{n}\left(x_{i}-\mu_{x}\right)^{2} \sum_{i=1}^{n}\left(y_{i}-\mu_{y}\right)^{2}\right]}
$$


Em que:

$\boldsymbol{x}$ : variável de entrada (a variável aleatória);

$\boldsymbol{y}$ : variável de saída (Função Estado Limite);

$\boldsymbol{\mu}_{\boldsymbol{x}}$ : média da variável $x$;

$\mu_{y}$ : média da variável $y$.

Para se chegar a uma redução das variáveis aleatórias a serem consideradas na análise de confiabilidade, vários grupos de variáveis foram estabelecidos, tendo como objetivo reunir em um mesmo grupo as variáveis que viessem a apresentar os maiores índices de importância. Calculou-se, então, o índice de confiabilidade $\beta$ para cada grupo.

\section{RESULTADOS}

\subsection{MÉTODO DE MONTE CARLO}

A partir da implementação do Método de Simulação de Monte Carlo, foram obtidos o índice de confiabilidade e a probabilidade de falha para a ponte em análise, descrita no item 4 . Os respectivos valores são apresentados na Tabela 4.

\section{TABELA 4: Resultados da análise de confiabilidade} por meio do método Monte Carlo.

\begin{tabular}{ccc} 
& $\mathbf{G}_{\mathbf{1}}$ & $\mathbf{G}_{\mathbf{2}}$ \\
\hline $\boldsymbol{\beta}$ & 4,40 & 2,21 \\
\hline $\boldsymbol{P}_{\boldsymbol{f}}$ & $5,41 \mathrm{E}-06$ & $1,36 \mathrm{E}-02$ \\
\hline & & FONTE: Autoria Própria
\end{tabular}

As Figuras 2 e 3 mostram a representação tridimensional do histograma Resistência versus Solicitação para as Funções Estado Limite $G_{1}$ e $G_{2}$, respectivamente, segundo os valores obtidos através do método de Monte Carlo. Em cada uma dessas figuras é possível observar um plano, o qual representa o respectivo plano de falha $G(x)=0$ da estrutura, e que divide a região segura da região de falha para cada estado limite considerado.

\subsection{MÉTODO FORM}

Implementando-se o método FORM, obtiveram-se os valores do ponto de projeto e do índice de confiabilidade $\beta$ em cada iteração, mostrados Nas Tabelas 5 e 6.

Dos resultados alcançados pode-se observar que os valores dos índices de confiabilidade calculados através do método Monte Carlo e através do FORM são próximos. Finalmente foram calculadas a probabilidade de falha $\left(P_{f}\right)$ e a confiabilidade $(C)$ das Funções Estado Limite $G_{1}$ e $G_{2}$, as quais são mostradas nas Tabelas 7 e 8 , respectivamente. A confiabilidade $(C)$ é dada pela Equação 30.

$$
C=1-P_{f}
$$

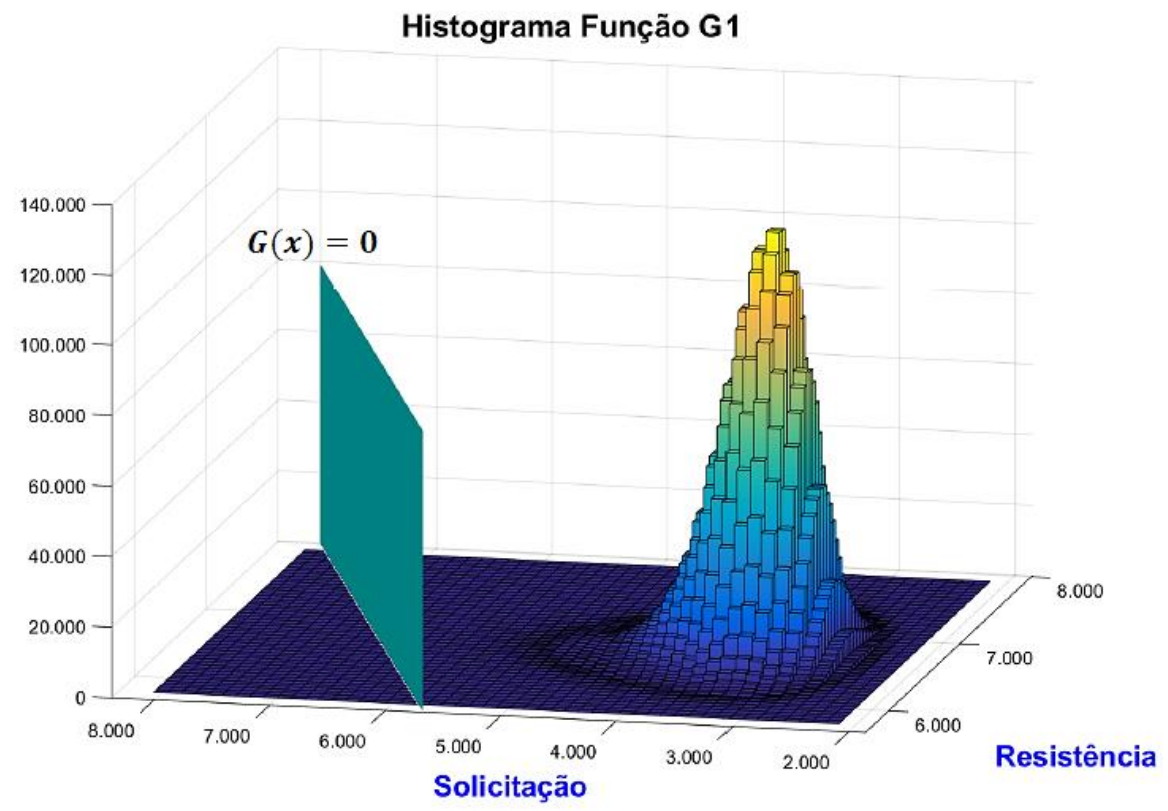

FIGURA 2: Histograma tridimensional Resistência x Solicitação da Função Estado Limite $G_{1}$, e plano de falha, obtidos através do programa MATLAB.

FONTE: Autoria própria. 


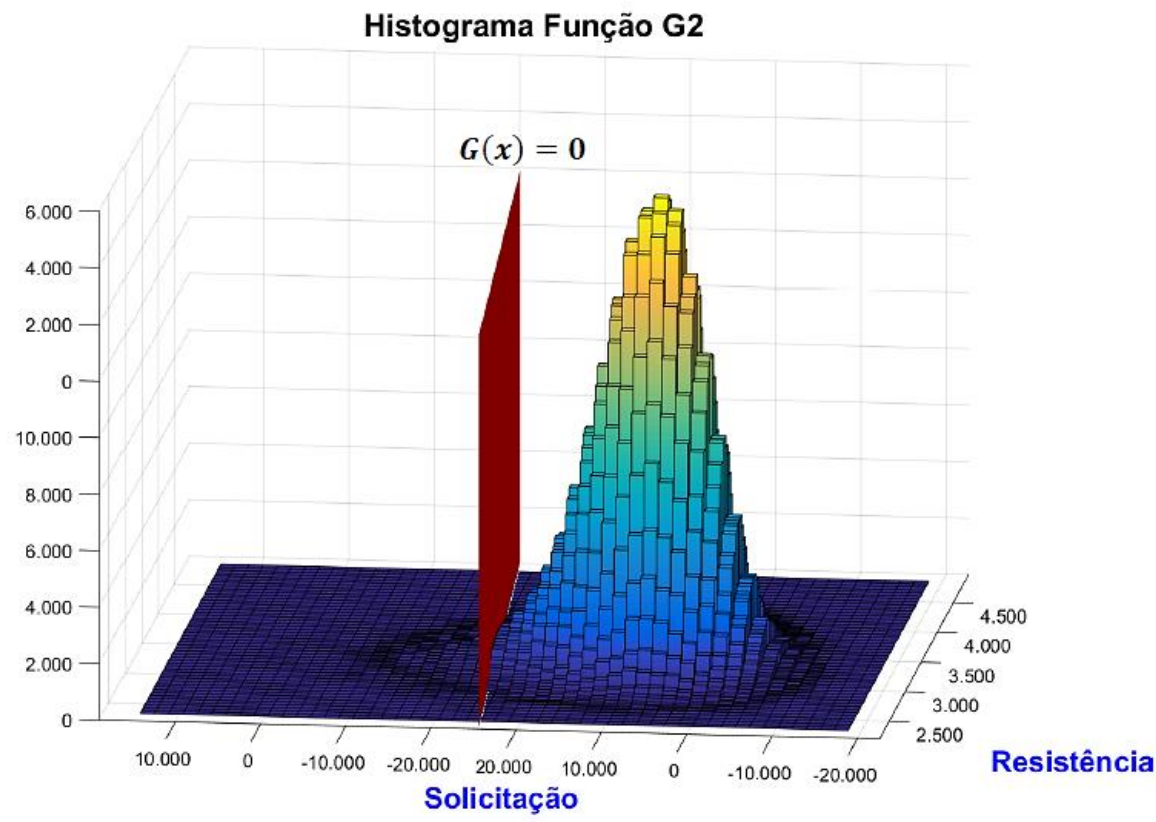

FIGURA 3: Histograma tridimensional Resistência x Solicitação da Função Estado Limite $G_{2}$, e plano de falha, obtidos através do programa MATLAB.

FONTE: Autoria própria.

\subsection{ANÁLISE DE SENSIBILIDADE}

Segundo o estabelecido no item 4, uma análise de sensibilidade foi efetuada utilizando dois métodos diferentes. Nas Tabelas 9 e 10 são apresentados os diferentes fatores de sensibilidade e índices de importância para cada variável aleatória, respectivamente. Na Tabela 11 são mostrados os coeficientes de Pearson para cada variável aleatória.

Nas Tabelas 12 e 13 são apresentados os valores do índice de confiabilidade $\beta$ para as funções $G_{1}$ e $G_{2}$, respectivamente, calculados no procedimento de redução das variáveis aleatórias para os diferentes casos. $O$ número de variáveis foi alterado até se alcançar o valor do índice de probabilidade obtido no item 5.1. Dos resultados apresentados, conclui-se que o número de variáveis aleatórias para a Função Estado Limite $G_{1}$ pode ser reduzido a seis $\left(M_{v e}, f_{p u}, h_{\text {viga }}, D C, A_{p s}\right.$ e $\left.f_{c}^{\prime}\right)$, e igualmente para a Função Estado Limite $G_{2}$ pode ser reduzido a seis $\left(M_{v e}, P_{\text {iniciall }}, D C, f_{p u}, f_{c}^{\prime}, y_{G c}\right)$.

TABELA 5: Valores das variáveis aleatórias em cada iteração no método FORM para a Função Estado Limite $\mathrm{G}_{1}$.

\begin{tabular}{cccccc} 
Variável/ Iteração & $\mathbf{1}$ & $\mathbf{2}$ & $\mathbf{3}$ & $\mathbf{4}$ & $\mathbf{5}$ \\
\hline $\boldsymbol{\beta}$ & 4,430 & 4,429 & 4,429 & 4,429 & 4,429 \\
\hline $\boldsymbol{A}_{\boldsymbol{p s}}\left[\mathbf{m}^{\mathbf{2}}\right]$ & 0,0027543 & 0,0027542 & 0,0027542 & 0,0027542 & 0,0027542 \\
\hline $\boldsymbol{y}_{\boldsymbol{b s}}[\mathbf{m}]$ & 0,103 & 0,103 & 0,103 & 0,103 & 0,103 \\
\hline $\boldsymbol{b}[\mathbf{m}]$ & 1,628 & 1,628 & 1,628 & 1,628 & 1,628 \\
\hline $\boldsymbol{f}_{\boldsymbol{c}}{ }_{\boldsymbol{c}}\left[\mathbf{k n} / \mathbf{m}^{\mathbf{2}}\right]$ & 44290,38 & 44270,89 & 44269,94 & 44269,92 & 44269,92 \\
\hline $\boldsymbol{f}_{\boldsymbol{p u}}\left[\mathbf{k n} / \mathbf{m}^{\mathbf{2}}\right]$ & 1942961,32 & 1942868,10 & 1942860,30 & 1942860,31 & 1942860,31 \\
\hline $\boldsymbol{h}_{\text {viga }}[\mathbf{m}]$ & 1,25 & 1,25 & 1,25 & 1,25 & 1,25 \\
\hline $\boldsymbol{D} \boldsymbol{C}[\mathbf{k N} / \mathbf{m}]$ & 17,09 & 17,11 & 17,11 & 17,11 & 17,11 \\
\hline $\boldsymbol{M}_{\boldsymbol{v e}}[\mathbf{k N} \cdot \mathbf{m}]$ & 5062,51 & 5059,47 & 5059,40 & 5059,40 & 5059,40 \\
\hline & & & & FONTE. Autoria Própria
\end{tabular}

FONTE: Autoria Própria. 
TABELA 6: Valores das variáveis aleatórias em cada iteração no método FORM para a Função Estado Limite $\mathrm{G}_{2}$.

\begin{tabular}{ccccccc} 
Variável/ Iteração & $\mathbf{1}$ & $\mathbf{2}$ & $\mathbf{3}$ & $\mathbf{4}$ & $\mathbf{5}$ & $\mathbf{6}$ \\
\hline $\boldsymbol{\beta}$ & 2,247 & 2,234 & 2,234 & 2,234 & 2,234 & 2,234 \\
\hline $\boldsymbol{A}_{\boldsymbol{p s}}\left[\mathbf{m}^{\mathbf{2}}\right]$ & 0,002764 & 0,002764 & 0,002764 & 0,002764 & 0,002764 & 0,002764 \\
\hline $\boldsymbol{y}_{\boldsymbol{b s}}[\mathbf{m}]$ & 0,102 & 0,102 & 0,102 & 0,102 & 0,102 & 0,102 \\
\hline $\boldsymbol{y}_{\boldsymbol{c c}}[\mathbf{m}]$ & 0,9503 & 0,9503 & 0,9503 & 0,9503 & 0,9503 & 0,9503 \\
\hline $\boldsymbol{f}_{\boldsymbol{c}}\left[\mathbf{k n} / \mathbf{m}^{\mathbf{2}}\right]$ & 44544,01 & 44512,60 & 44509,18 & 44508,86 & 44508,83 & 44508,83 \\
\hline $\boldsymbol{f}_{\boldsymbol{p u}}\left[\mathbf{k n} / \mathbf{m}^{2}\right]$ & 1970039,94 & 1970097,92 & 1970103,15 & 1970103,65 & 1970103,69 & 1970103,70 \\
\hline $\boldsymbol{P}_{\text {inicial }}[\mathbf{m}]$ & 4133,08 & 4122,39 & 4121,39 & 4121,30 & 4121,29 & 4121,29 \\
\hline $\boldsymbol{D C}[\mathbf{k N} / \mathbf{m}]$ & 16,97 & 17,02 & 17,03 & 17,03 & 17,03 & 17,03 \\
\hline $\boldsymbol{M}_{\text {ve }}[\mathbf{k N} \cdot \mathbf{m}]$ & 2636,20 & 2618,62 & 2616,98 & 2616,83 & 2616,81 & 2616,81 \\
\hline
\end{tabular}

FONTE: Autoria Própria.

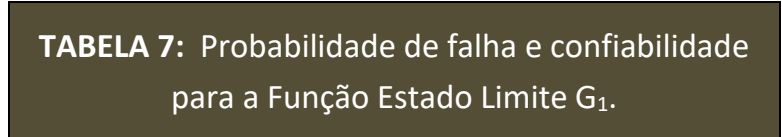

\begin{tabular}{ccc} 
& FORM & MONTE CARLO \\
\hline $\boldsymbol{P}_{\boldsymbol{f}}$ & $4,74 \mathrm{E}-06$ & $5,41 \mathrm{E}-06$ \\
\hline $\boldsymbol{C}$ & 0,99999526 & 0,999994587 \\
\hline & & FONTE: Autoria Própria.
\end{tabular}

TABELA 8: Probabilidade de falha e confiabilidade para a Função Estado Limite $\mathrm{G}_{2}$.

\begin{tabular}{ccc} 
& FORM & MONTE CARLO \\
\hline $\boldsymbol{P}_{\boldsymbol{f}}$ & $1,27 \mathrm{E}-02$ & $1,36 \mathrm{E}-02$ \\
\hline $\boldsymbol{C}$ & 0,98727 & 0,98645 \\
\hline & & FONTE: Autoria Própria.
\end{tabular}

\begin{tabular}{|c|c|c|c|}
\hline \multicolumn{2}{|c|}{$\alpha_{i}$ para $G_{1}$} & \multicolumn{2}{|c|}{$\alpha_{i}$ para $G_{2}$} \\
\hline$A_{p s}$ & $6,13 \mathrm{E}-02$ & $A_{p s}$ & $8,72 \mathrm{E}-03$ \\
\hline$y_{b s}$ & $2,15 \mathrm{E}-02$ & $y_{b s}$ & $2,13 \mathrm{E}-03$ \\
\hline$b$ & $1,12 \mathrm{E}-02$ & $y_{G c}$ & $1,72 \mathrm{E}-02$ \\
\hline$f_{c}^{\prime}$ & $1,58 \mathrm{E}-02$ & $f_{c}^{\prime}$ & $1,51 \mathrm{E}-02$ \\
\hline$f_{p u}$ & $1,22 \mathrm{E}-01$ & $f_{p u}$ & $7,00 \mathrm{E}-03$ \\
\hline$h_{\text {viga }}$ & $4,19 \mathrm{E}-02$ & $P_{\text {inicial }}$ & $3,09 \mathrm{E}-01$ \\
\hline$D C$ & $1,08 \mathrm{E}-01$ & $D C$ & $1,92 \mathrm{E}-01$ \\
\hline$M_{v e}$ & $9,83 \mathrm{E}-01$ & $M_{v e}$ & $9,31 \mathrm{E}-01$ \\
\hline
\end{tabular}

FONTE: Autoria Própria. 


\begin{tabular}{|c|c|c|c|}
\hline \multicolumn{2}{|c|}{$I_{i}$ para $\mathbf{G}_{\mathbf{1}}$} & \multicolumn{2}{|c|}{$I_{i}$ para $\mathbf{G}_{\mathbf{2}}$} \\
\hline$A_{p s}$ & 3,76E-03 & $A_{p s}$ & 7,60E-05 \\
\hline$y_{b s}$ & $4,60 \mathrm{E}-04$ & $y_{b s}$ & $4,55 \mathrm{E}-06$ \\
\hline$b$ & $1,26 \mathrm{E}-04$ & $y_{G c}$ & 2,97E-04 \\
\hline$f_{c}^{\prime}$ & $2,49 \mathrm{E}-04$ & $f_{c}^{\prime}$ & $2,29 \mathrm{E}-04$ \\
\hline$f_{p u}$ & $1,49 \mathrm{E}-02$ & $f_{p u}$ & 4,90E-05 \\
\hline$h_{\text {viga }}$ & $1,75 \mathrm{E}-03$ & $P_{\text {inicial }}$ & $9,53 \mathrm{E}-02$ \\
\hline$D C$ & 1,17E-02 & $D C$ & $3,70 \mathrm{E}-02$ \\
\hline$M_{v e}$ & 9,67E-01 & $M_{v e}$ & 8,67E-01 \\
\hline
\end{tabular}

\begin{tabular}{|c|c|c|c|}
\hline \multicolumn{2}{|c|}{$r_{i}$ para $\mathbf{G}_{1}$} & \multicolumn{2}{|c|}{$r_{i}$ para $\mathbf{G}_{2}$} \\
\hline$A_{p s}$ & 1,86E-01 & $A_{p s}$ & $-1,46 \mathrm{E}-02$ \\
\hline$y_{b s}$ & $-6,55 E-02$ & $y_{b s}$ & $3,99 \mathrm{E}-03$ \\
\hline$b$ & $3,52 \mathrm{E}-02$ & $y_{G c}$ & $-4,88 \mathrm{E}-03$ \\
\hline$f_{c}^{\prime}$ & 1,01E-01 & $f_{c}^{\prime}$ & $3,93 \mathrm{E}-02$ \\
\hline$f_{p u}$ & $3,73 \mathrm{E}-01$ & $f_{p u}$ & $-1,08 \mathrm{E}-02$ \\
\hline$h_{\text {viga }}$ & 1,28E-01 & $P_{\text {inicial }}$ & $5,10 \mathrm{E}-01$ \\
\hline$D C$ & $-3,25 E-01$ & $D C$ & $-3,17 \mathrm{E}-01$ \\
\hline$M_{v e}$ & $-8,30 \mathrm{E}-01$ & $M_{v e}$ & $-7,99 E-01$ \\
\hline
\end{tabular}

\section{CONCLUSÕES}

No presente trabalho, um procedimento para avaliar a segurança de pontes em concreto protendido foi desenvolvido e sistematizado. Estabeleceu-se como objetivo a obtenção da probabilidade de falha $P_{f}$ e do índice de confiabilidade $\beta$ para uma ponte de concreto protendido em um sistema viga-e-laje.

A avaliação de segurança de uma ponte de concreto protendido, realizada pelo procedimento sistematizado proposto, permitiu concluir que o método de simulação Monte Carlo, implementado através do programa MATLAB consegue proporcionar uma resposta da probabilidade de falha muito próxima à aquela que seria obtida através de um método analítico, permitindo avaliar a segurança da estrutura de uma forma menos complexa.

A análise de sensibilidade realizada apontou as variáveis que exercem maior influência no cálculo da probabilidade de falha: a capacidade última à flexão de uma viga em concreto protendido é sensível à área $A_{p s}$ e à resistência $f_{p u}$ da armadura de protensão, e à altura da viga $h_{\text {viga }}$, como também observado por Chandrasekar e Dayaraatnam (1975), Tabsh e Nowak (1991) e Rakoczy e Nowak (2013); a tensão à tração do concreto é sensível à força aplicada nos cabos de protensão $P_{\text {inicial, }}$ ao momento devido ao veículo de projeto $M_{v e}$ e ao peso próprio dos elementos estruturais $D C$.

O presente trabalho reuniu, em um procedimento numérico, as etapas para a avaliação da segurança de pontes em concreto protendido. A validade do procedimento foi verificada por meio de comparação com método analítico e com valores de probabilidade de falha encontrados na literatura corrente. A análise de sensibilidade permitiu apontar quais as variáveis aleatórias exercem maior influência na segurança desse tipo de estrutura. 
TABELA 12: Índice de confiabilidade e probabilidade de

falha para $\mathrm{G}_{1}$ quando o número de variáveis aleatórias é

alterado.

\begin{tabular}{|c|c|c|}
\hline Variáveis Aleatórias & $\boldsymbol{\beta}$ & $\boldsymbol{P}_{f}$ \\
\hline$M_{v e}$ & 4,3 & $8,540 \mathrm{E}-06$ \\
\hline$M_{v e}, f_{p u}$ & 4,5 & $3,092 \mathrm{E}-06$ \\
\hline$M_{\text {ve, }} f_{\text {pu }}, h_{\text {viga }}$ & 4,61 & $2,013 \mathrm{E}-06$ \\
\hline$M_{v e,} f_{\text {pu, }} h_{\text {viga }}, D C$ & 4,34 & 7,124E-06 \\
\hline$M_{v e,} f_{p u}, h_{v i g a}, D C, f_{c}^{\prime}$ & 4,42 & $4,935 \mathrm{E}-06$ \\
\hline$M_{v e,} f_{p u}, h_{v i g a} D C, A_{p s}$ & 4,32 & $7,801 \mathrm{E}-06$ \\
\hline$M_{v e,} f_{p u}, h_{v i g a}, D C, A_{p s,} f_{c}^{\prime}$ & 4,4 & $5,413 \mathrm{E}-06$ \\
\hline
\end{tabular}

TABELA 13: Índice de confiabilidade e probabilidade de falha para $\mathrm{G}_{2}$ quando o número de variáveis aleatórias é alterado.

\begin{tabular}{|c|c|c|}
\hline Variáveis Aleatórias & $\beta$ & $\boldsymbol{P}_{f}$ \\
\hline$M_{\text {ve, }} P_{\text {inicial }}$ & 2,34 & $9,642 \mathrm{E}-03$ \\
\hline$M_{\text {ve, }} P_{\text {inicial }} D C$ & 2,20 & $1,390 \mathrm{E}-02$ \\
\hline$M_{v e,} P_{\text {inicial }} D C, f_{p u}$ & 2,19 & $1,426 \mathrm{E}-02$ \\
\hline$M_{\text {ve, }}, P_{\text {inicial, }} D C, f_{p u}, f_{c}^{\prime}$ & 2,215 & $1,338 \mathrm{E}-02$ \\
\hline$M_{\text {ve, }} P_{\text {inicial }} D C, f_{p u} f_{c}^{\prime}, y_{G c}$ & 2,21 & $1,355 \mathrm{E}-02$ \\
\hline
\end{tabular}

\section{AGRADECIMENTOS}

Os autores agradecem à CAPES pelo apoio financeiro fornecido para o desenvolvimento da presente pesquisa.

\section{REFERÊNCIAS BIBLIOGRÁFICAS}

ABEJIDE, O. S. Safety analysis of prestressed concrete bridge beams in flexure. International Journal of Sciences, Vol. 17, n. 2, 2014, 260-274 p.

AGRAWAL, G., BHATTACHARYA, B. Partial safety factor design of rectangular partially prestressed concrete beams in ultimate flexural limit state. Journal of Structural Engineering, Vol. 37, n. 4, 2010, 257-267 p.

Al-HARTHY, A. S., FRANGOPOL, D. M. Reliability assessment of prestressed concrete beams. Journal of Structural Engineering, ASCE, Vol. 120, n. 1, 1994, 180$199 \mathrm{p}$.
AKGÜL, F., FRANGOPOL, D. M. Lifetime performance analysis of existing prestressed concrete bridge superstructures. Journal of Structural Engineering, Vol. 130 , n. $12,2004,1889-1903$ p.

AMERICAN ASSOCIATION OF STATE HIGHWAY AND TRANSPORTATION OFFICIALS (AASHTO). AASHTO LRFD Bridge Design Specifications, Customary U.S. Units, 6th Edition, 2012, 1661 p.

BECK A. T. Confiabilidade estrutural. Apostila de Aula USP, Sao Carlos, 2006, 182 p.

BIONDINI, F., BONTEMPI, F., FRANGOPOL, D. M. Reliability of material and geometrically nonlinear reinforced and prestressed concrete structures. Computational Fluid and Solid Mechanics, Vol. 82, 2004, 2208-2212 p.

CHANDRASEKAR P., DAYARAATNAM P. Analysis of probability of failure of prestressed concrete beams. Building Science, Vol. 10, 1975, 161-167 p. 
CHENG, J., CAI, C. S., XIAO, R. C. Probabilistic response analysis of cracked prestressed concrete beams. Advances in Structural Engineering, Vol. 10, n. 1, 2007, 1$10 \mathrm{p}$.

GARDNER, R. H. et al. A comparison of sensitivity analysis and error analysis based on a stream ecosystem model. Ecological Modelling, Vol. 12, n. 3, 1981, 173-190 p.

HALHALLI, V. Reliability index for post-tensioned prestressed concrete girders in flexure based on IS: 1343-1980 Provisions. International Journal of Engineering Innovation \& Research, Vol. 3, n. 4, 2014.

HAMBY, D. M. A review of techniques for parameter sensitivity analysis of environmental models. Environmental monitoring and assessment, Vol. 32, n. 2, 1994, 135-154 p.

JACINTO, L. Avaliação da segurança de pontes existentes - abordagem probabilística Bayesiana. Tese de doutorado, Universidade de Nova de Lisboa, 2011.

JCSS. Guideline for reliability based assessment of existing structures. The Joint Committee in Structural Safety, January, 2000.

NOWAK, A. S., COLLINS, K. R. Reliability of structures. CRC Press, 2000.

NOWAK, A. S., PARK, C. H., CASAS, J. R. Reliability analysis of prestressed concrete bridge girders: comparison of Eurocode, Spanish Norma IAP and AASHTO LRFD. Structural Safety, Vol. 23, n. 4, 2001, 331-344 p.

RACKWITZ R., FIESSLER, B. Structural reliability under combined random load sequences. Computer and Structures, Vol. 9, 1978, 484-494 p.

RAKOCZY, A. M., NOWAK, A. S. Reliability-based sensitivity analysis for prestressed concrete girder bridges. $\mathrm{PCl}$ journal, 2013, Vol. 58, n.4.

ROCHA, R. G. et al. Análise de confiabilidade de vigas portuárias de concreto protendido. ENGEVISTA, Vol. 17, n. 4, 2015, 573-587 p.

SAN MARTINS, D. A. Confiabilidade de vigas prétracionadas de concreto protendido. Dissertação de Mestrado, Universidade Federal do Rio Grande do Sul, 2014.

STEINBERG, E. Structural reliability of prestressed UHPC flexure models for bridge girders. Journal of Bridge Engineering, Vol. 15, n. 1, 2010, 65-72 p.

TABSH, B. S. W., NOWAK, A. S. Reliability of highway girder bridges. Journal of Structural Engineering, Vol. 117 , n. 8, 1991, 2372-2388 p. 